\title{
Effect of Naloxone-3-Glucuronide and $N$-Methylnaloxone on the Motility of the Isolated Rat Colon After Morphine
}

\author{
Peter Reber • Rudolf Brenneisen - Beatrice Flogerzi • \\ Catarina Batista • Peter Netzer • Ulrich Scheurer
}

Received: 3 March 2006 / Accepted: 4 August 2006 / Published online: 9 January 2007

(C) Springer Science+Business Media, Inc. 2006

\begin{abstract}
The effect of the opioid antagonists naloxone3 -glucuronide and $\mathrm{N}$-methylnaloxone on rat colon motility after morphine stimulation was measured. The rat model consisted of the isolated, vascularly perfused colon. The antagonists $\left(10^{-4} \mathrm{M}\right.$, intraluminally) and morphine $\left(10^{-4} \mathrm{M}\right.$, intra-arterially) were administered from 20 to 30 and from 10 to $50 \mathrm{~min}$, respectively. Colon motility was determined by the luminal outflow. The antagonist concentrations in the luminal and venous outflow were measured by high-performance liquid chromatography. Naloxone-3glucuronide and $N$-methylnaloxone reversed the morphineinduced reduction of the luminal outflow to baseline within 10 and $20 \mathrm{~min}$, respectively. These antagonists were then excreted in the luminal outflow and could not be found in the venous samples. Naloxone, produced by hydrolysis or demethylation, was not detectable. In conclusion, highly polar naloxone derivatives peripherally antagonize the motilitylowering effect of morphine in the perfused isolated rat colon, are stable, and are not able to cross the colon-mucosal blood barrier.
\end{abstract}

\section{P. Reber}

Department of Vascular Surgery, Lindenhof Hospital, Bern, Switzerland

R. Brenneisen $(\bowtie) \cdot$ C. Batista

Department of Clinical Research, University of Bern, Murtenstrasse 35,

CH-3010 Bern, Switzerland

e-mail: rudolf.brenneisen@dkf.unibe.ch

B. Flogerzi · P. Netzer · U. Scheurer

Clinic for Gastroenterology, University Hospital,

Bern, Switzerland
Keywords Isolated rat colon $\cdot$ Morphine $\cdot$ Motility inhibition · Naloxone-3-glucuronide $\cdot N$-Methylnaloxone Opioid receptor antagonists

\section{Introduction}

Opioid therapy is the mainstay of treatment for cancer-related pain affecting approximately 9 million people worldwide annually [1], and 55 to $95 \%$ of patients with advanced cancer have severe pain [2]. Morphine (MO) is the drug of choice for the management of moderate to severe chronic cancer pain because of its effectiveness, tolerability, ease of use, and low cost [3]. Unfortunately, constipation is one of the most troublesome side effects of opioid therapy and often adds to the distress of patients being treated for chronic pain [4]. It is even a problem when opiates are administered on a short-term basis for postoperative pain relief [5]. In addition to constipation, patients receiving opioids have other aspects of opioid bowel dysfunction, including lower abdominal discomfort, fecal impaction with overflow diarrhea and incontinence, nausea, vomiting, and inadequate absorption of oral drugs [6]. Furthermore, it has been demonstrated that functional deterioration in patients with advanced cancer correlates closely with poor bowel function [7].

Whereas the analgesic action of opioids is mediated through the central nervous system, constipation is a consequence of their action on the gut wall, reducing intestinal secretion and motility in both small and large bowel, with a lengthening of gut transit time [8]. These adverse gastrointestinal opioid effects are produced through the activation of central and peripheral $\mu$-opioid receptors $[9,10]$. The activation of peripheral receptors within the gastrointestinal tract appears to be particularly important because the magnitude of bowel dysfunction correlates more closely with opioid 
concentrations in the enteric nervous system than with concentrations in the central nervous system [11]. Similarly, selective activation of gastrointestinal $\mu$-opioid receptors by poorly absorbed $\mu$-opioid agonists, such as loperamide, results in profound inhibition of gastrointestinal transit [12]. The adverse effects of opioids on gastrointestinal function can be reversed by antagonism of $\mu$-receptors with currently available $\mu$-opioid antagonists such as naloxone (NX) and nalmefene. However, these antagonists are centrally active, therefore they also antagonize analgesia and precipitate symptoms of opioid withdrawal in MO-dependent subjects.

The development of $\mu$-opioid receptor antagonists with an action restricted to the periphery is therefore necessary to prevent the effects of opioids on the gastrointestinal tract without antagonizing the central effects, such as analgesia, and producing opioid withdrawal symptoms.

In humans NX undergoes an extensive first-pass liver effect with formation of the main metabolite naloxone-3glucuronide (NXG). In contrast to morphine-glucuronides [13-15], the binding affinity and potency of NXG for opioid receptors are not known. Whereas NX is easily absorbed, we hypothesize that the highly polar NXG should not reach the systemic circulation and, therefore, not reduce analgesia. A similar pharmacokinetic behavior is assumed by $N$ methylnaloxone (MNX), like NXG, a peripherally acting opioid receptor antagonist [16-20].

The aim of this study was to investigate in an isolated, arterially perfused rat colon whether intraluminally (i.1.) administered NXG and MNX are able to normalize MO-delayed colon transit without being absorbed into the systemic circulation.

\section{Methods}

Preparation of the isolated perfused rat colon

The model of an isolated, vascularly perfused rat colon has been described in detail elsewhere [21, 22]. Each colon preparation was used just for one perfusion trial. Briefly, adult Wistar rats of either sex weighing 250-300 g were used. They were cared for in accordance with laboratory animal care and use guidelines established by the Veterinary Services of the Canton of Bern (animal assay permit no. 83/00). Before operation, rats were fasted for $24 \mathrm{hr}$, with free access to water. The animals were anesthesized with pentobarbital, $50 \mathrm{mg} \mathrm{kg}{ }^{-1}$ body weight intraperitoneally, before midline laparotomy. The hindgut and the upper small intestine were freed up to the duodenum and separated after ligation of the supplying vessels.

A silastic tube was inserted into the cecum. Similarly, the distal descending colon was opened by a transverse inci- sion, and the lumen was flushed with $20 \mathrm{ml}$ of prewarmed isotonic saline from the proximal end before a second silastic tube was inserted into the distal descending colon. Subsequently, the inferior mesenteric artery was divided distally of the left colic artery. Finally, the distal descending colon was transected. At this point, the canulated colon (i.e., cecum, ascending, transversing, and descending colon) receives its arterial blood supply only from the superior mesenteric artery. The superior mesenteric artery and the superior mesenteric vein were next dissected and canulated with polyethylene tubes (OD, 0.067 in.; Intramedic polyethylene tubing, Becton Dickinson, Parsippany, NJ). Vascular perfusion was started immediately after cannulation of the superior mesenteric artery at a perfusion rate of $3 \mathrm{ml} \mathrm{min}-1$ (Gibson Miniplus 3; Abimed, UK) to avoid any ischemia of the colon. Finally, the canulated colon was freed from the abdominal cavity by dividing the posterior attachments. Vascular and luminal perfusion (LKB Microperpex Peristaltic Pump Model. 2132; Pharmacia Biotech, Sweden) of the colon was performed in a thermostatically controlled perfusion chamber (GFL Laborbedarf, Burgwedel, Germany) [23]. Luminal perfusion was performed at a starting perfusion rate of $0.75 \mathrm{ml} \mathrm{min}^{-1}$, resulting in an perfusion pressure of $60 \pm 5 \mathrm{~mm} \mathrm{Hg}$ at the beginning of the experiments and in the control animals. There was no apparent distention of the colon at this perfusion rate. The luminal perfusion pressure was continuously measured and kept constant by altering the luminal perfusion rate if necessary. The luminal outflow represented the sum of motility actions, secretions, and resorptions of the isolated perfused colon. Therefore we assumed that the luminal outflow reflected colon motility. The vascular and luminal perfusion fluid (modified Krebs-RingerHenseleit solution) used was isosmolar with rat plasma and had the following composition $(\mathrm{mM}): \mathrm{NaCl}, 125 ; \mathrm{KCl}$, 4.3; $\mathrm{MgCl}_{2}, 1 ; \mathrm{CaCl}_{2}, 2.5 ; \mathrm{NaHCO}_{3}, 25 ; \mathrm{NaH}_{2} \mathrm{PO}_{4}, 1$; and glucose, 5. The perfusion fluid was freshly prepared, filtered through Schleicher \& Schuell 597 filter paper (Dassel, Germany) before use, had a $\mathrm{pH}$ of 7.4 , and was bubbled with $\mathrm{O}_{2}(95 \%)$ and $\mathrm{CO}_{2}$ (5\%; Evaporator Type 2362; Liebich, Bielefeld, Germany). The temperature was maintained at $37^{\circ} \pm 0.3^{\circ} \mathrm{C}$. Viability of each colon was tested at the end of the experiment by its ability to contract after a mechanical stimulus.

Colon motility and outflow measurements

The agonist solution consisted of $10^{-4} \mathrm{M} \mathrm{MO}$ (morphine hydrochloride trihydrate Ph. Eur.; Merck, provided by Grogg, Bern, Switzerland) dissolved in perfusion fluid. This concentration resulted from a preceding dose-finding study with $10^{-6}, 10^{-5}$, and $10^{-4} \mathrm{M}$ MO. The solution was applied intra-arterially (i.a.) from 10 to $50 \mathrm{~min}$ after T0 (=time point of steady state). The equimolar antagonist solutions 
were prepared by dissolving $10^{-4} \mathrm{M} \mathrm{NXG}$ (naloxone-3- $\beta$-Dglucuronide hydrate; Lipomed, Arlesheim, Switzerland) or $10^{-4}$ M MNX (naloxone methiodide; Sigma-Aldrich, Buchs, Switzerland) in perfusion fluid. Perfusion fluid was used as placebo solution. NXG $(n=9)$, MNX $(n=6)$, and placebo $(n=3)$ solutions were applied intraluminally (i.l.) from 20 to 30 min after T0. Luminal and venous outflow samples were collected during 5-min intervals starting $5 \mathrm{~min}$ before (baseline) and 5, 15, 25, 35, and $45 \mathrm{~min}$ after the administration of MO. The luminal and venous outflows were determined by weighing. There was no significant difference between arterial inflow and venous outflow, indicating the absence of significant resorption (data not shown).

Determination of NX, NXG, and MNX in the luminal and venous outflow by HPLC

An ASPEC XL robotic system (Gilson, Villiers Le Bel, France) was used for extraction of the biological samples. To $1 \mathrm{ml}$ luminal outflow, diluted 1:10 with perfusion fluid and filtrated, or $1 \mathrm{ml}$ venous outflow, undiluted and filtrated, $100 \mu \mathrm{l}$ internal standard solution (1.5 mg ethylmorphine hydrochloride $\mathrm{Ph}$. Eur. [Merck], dissolved in $100 \mathrm{ml}$ bidistillated water) and $3.0 \mathrm{ml} 0.5 \mathrm{M}$ carbonate buffer, $\mathrm{pH} 9.3$, were added. After vortexing, the mixture was applied to solid phase extraction (SPE) columns (Chromabond C-18 ec, 3 ml, $500 \mathrm{mg}$; Macherey-Nagel, Oensingen, Switzerland), preconditioned with two aliquots of $2.5 \mathrm{ml}$ methanol, $40 \%$ acetonitrile in $0.01 \mathrm{M}$ phosphate buffer $\mathrm{pH} 2.1(\mathrm{v} / \mathrm{v})$, and bidistillated water, respectively. After washing with six aliquots of $2.5 \mathrm{ml}$ $0.005 \mathrm{M}$ carbonate buffer, $\mathrm{pH} 9.3,0.5 \mathrm{ml}$ water, and $0.5 \mathrm{ml}$ $40 \%$ acetonitrile in $0.01 \mathrm{M}$ phosphate buffer, $\mathrm{pH} 2.1(\mathrm{v} / \mathrm{v})$, elution was performed with three aliquots of $1.0 \mathrm{ml} 70 \%$ acetonitrile in $0.01 \mathrm{M}$ phosphate buffer, $\mathrm{pH} 2.1(\mathrm{v} / \mathrm{v})$. The eluate was then concentrated at $45^{\circ} \mathrm{C}$ under $\mathrm{N}_{2}$ to dryness, redissolved in $120 \mu \mathrm{l}$ HPLC mobile phase A (see below), sonicated, and filtrated. Eight microliters of the SPE extracts was then used for HPLC analysis. An Agilent 1100 Series Capillary HPLC System (Agilent Technologies, Waldbronn, Germany) was used. The extracts were separated at $45^{\circ} \mathrm{C}$ on a Zorbax XDB C-8 3- $\mu$ m column (Agilent Technologies; $150 \times 0.5 \mathrm{~mm}$ i.d.) using a multistep gradient (mobile phase A, bidistilled water $+0.05 \%$ trifluoroacetic acid $[\mathrm{v} / \mathrm{v}] ; \mathrm{B}$, acetonitrile $+0.05 \%$ trifluoroacetic acid; $3 \%$ B [0-6 min], 3\%-12\% B [6-14 $\mathrm{min}$ ], 12\%-20\% B [14-20 min], 20\%$100 \%$ B [20-21 min], $100 \%$ B [21-30 min], 100\%-3\% B [30-35 min], 3\% B [35-45 min]; flow rate of $15 \mu 1 \mathrm{~min}^{-1}$ ). Quantitation of NX, NXG, and MNX was performed at 205 $\mathrm{nm}$ (diode array detector) based on peak areas and using the internal standard method. Validation data were as follows: SPE extraction efficiency, 66-91\%; calibration graph, linear in the range of 50-9000 $\mathrm{ng} \mathrm{ml}^{-1}, r^{2}>0.993$; intra- and interassay precision, $\pm 1.2 \%-7.0 \% \mathrm{RSD}$; intra- and inter- assay accuracy, $-1.8 \%$ to $-1.3 \%$; limits of quantitation and detection, 20 and $10 \mathrm{ng} \mathrm{ml}^{-1}$, respectively (signal-to-noise ratio, 5:1).

\section{Statistics}

Statistical analysis was performed with SPSS version 11 (Mac) using the Mann-Whitney test (nonparametric). Significance was assessed at the $P<0.05$ level (twotailed).

\section{Results}

Effect of NXG on the isolated rat colon motility inhibited by MO

Figure 1 shows that the luminal outflow of the isolated, perfused rat colon was reduced by $10^{-4} \mathrm{M}$ i.a. MO, from $7.74 \pm 0.41$ to $5.10 \pm 0.32 \mathrm{~g}$ (mean $\pm \mathrm{SEM} ; n=9$ ) within $10 \mathrm{~min}$. Therefore, $20 \mathrm{~min}$ after T0 the colon motility was decreased to $66.8 \% \pm 2.2 \%$ of the baseline value. With a luminal outflow of $7.62 \pm 0.48 \mathrm{~g}(P=0.009)$, this colon motilityinhibiting effect was, from 20 to $30 \mathrm{~min}$, almost reversed to baseline by $10^{-4} \mathrm{M}$ i.l. NXG. The MO effect reappeared 10 min after stopping the NXG administration. When using placebo (perfusion fluid; $n=3$ ) the colon motility-inhibiting effect by MO was not much influenced between 20 and 40 min. After 50 min the outflow was similar for NXG and placebo.

Effect of MNX on the isolated rat colon motility inhibition by $\mathrm{MO}$

The luminal outflow was reduced by $10^{-4} \mathrm{M}$ i.a. MO from $6.73 \pm 0.31$ to $4.06 \pm 0.24 \mathrm{~g}$ (mean $\pm \mathrm{SEM} ; n=6$ ) within $10 \mathrm{~min}$ (Fig. 2), corresponding to $60.4 \% \pm 2.7 \%$ of the baseline motility. Fourty minutes after T0, $10^{-4} \mathrm{M}$ i.l. MNX increased the luminal outflow to $7.53 \pm 0.28 \mathrm{~g}$ $(P=0.02)$, corresponding to $97.7 \% \pm 4.6 \%$ of the baseline value.

NXG and MNX levels in the luminal and

venous outflow of the isolated rat colon

In the luminal outflow samples collected after i.l. NXG, the NXG concentrations measured by a fully validated HPLC method ranged from 0 (baseline) to $88.38 \mu \mathrm{g} \mathrm{ml}^{-1}$. In the venous outflow no NXG and NX could be detected. After i.l. MNX, the luminal MNX concentrations varied between 0 (baseline) and $33.69 \mu \mathrm{g} \mathrm{ml}^{-1}$. Again, no MNX was detectable in the venous outflow. 
Fig. 1 Luminal outflow of the isolated, perfused rat colon after i.a. administration of $10^{-4} \mathrm{M}$ MO from 10 to $50 \mathrm{~min}, 10^{-4} \mathrm{M}$ i.l. NXG from 20 to $30 \mathrm{~min}$ $(\bullet ; n=9)$, and $10^{-4} \mathrm{M}$ i.l. placebo (perfusion fluid) from 20 to $30 \min (\Lambda ; n=3)$. The graphs show the reversal effect of the opioid antagonist NXG on the MO-stimulated colon motility inhibition vs. placebo. * Significant at $P<0.05$ vs. placebo

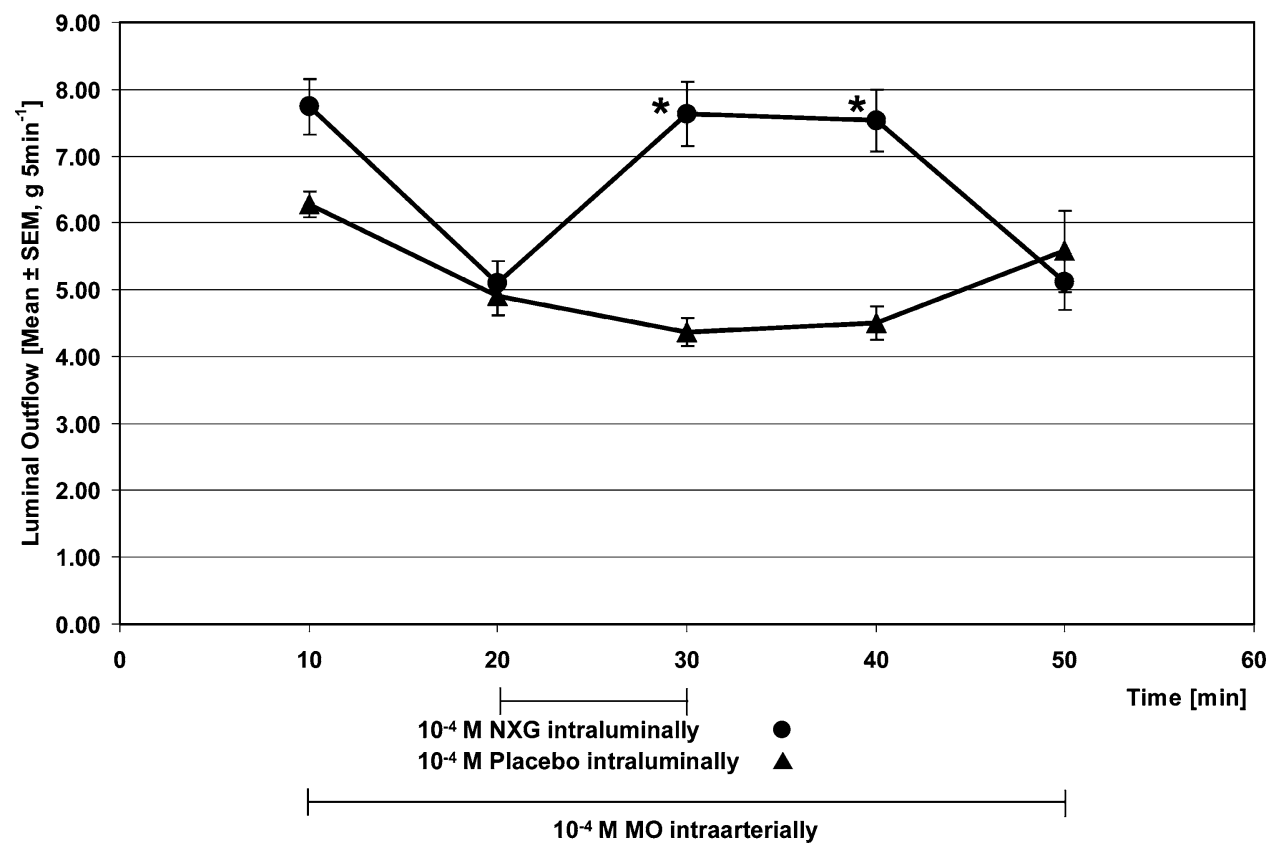

\section{Discussion}

The ex vivo model of the isolated, vascularly perfused rat colon used in this study proved to be suitable and robust for measuring agonistic and antagonistic effects of opioids on the colonic motility. In a dose-finding study, micromolar concentrations of MO resulted in the best organ response. In addition, these rather high concentrations are in accordance with those used in previous opioid experiments on isolated rat colons $[24,25]$ as well as guinea pig ileal circular muscle [26].
To the best of our knowledge, the present data show, for the first time, that the opioid glucuronide NXG exerts a peripheral effect on colon motility similar to that of the quarternary opioid MNX. In contrast to placebo, NXG and MNX administered i.l. significantly reversed the effects of i.a. MO on colonic motility. Neither NXG or MNX given i.l. nor NX could be detected in the venous outflow of the organ preparation. These findings suggest that the highly polar opioid antagonists are not able to pass the colonic mucosal blood barrier. Furthermore, the absence of NX in the venous outflow indicates that NXG and MNX are luminally not hydrolyzed and demethylated, respectively, by
Fig. 2 Luminal outflow of the isolated, perfused rat colon after i.a. administration of $10^{-4} \mathrm{M}$ MO from 10 to $50 \mathrm{~min}$ and of $10^{-4} \mathrm{M}$ i.l. MNX from 20 to $30 \min (n=6)$. The graph shows the reversal effect of the opioid antagonist MNX on the MO-stimulated colon motility inhibition. ${ }^{*}$ Significant at $P<$ 0.05 vs. placebo

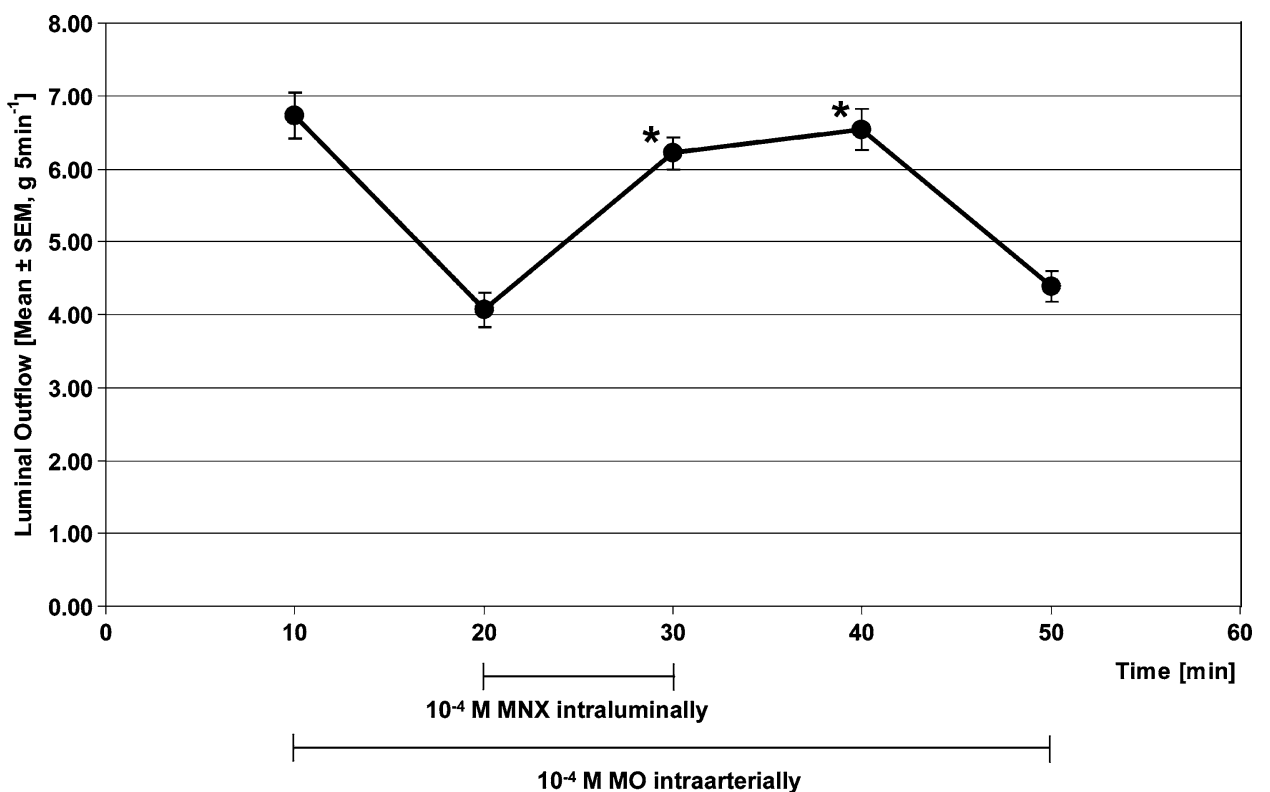


enzymes or bacteria to the easily absorbable NX. The latter phenomenon has been described for chronic pain patients treated with MO, where NX reduced constipation but caused opioid withdrawal symptoms [27]. This indicates a central effect of NX despite the well-known first-pass glucuronidation of NX in the liver. Similar observations were made in advanced cancer patients, where NX at doses $>20 \%$ of the 24-hr dose of MO had a marked laxative effect but also produced opioid withdrawal signs in some of the patients [28]. Further studies were postulated evaluating the ideal dose of oral NX which exerts the peripheral laxative effect without producing withdrawal symptoms.

In rats where oral NX reversed the constipating effects of oral MO, no influence on nociception was observed [29]. It was concluded that an extensive metabolization of NX is responsible for the lack of central activity.

Our results support the findings that in MO-dependent rats, peroral NXG induced diarrhea without signs of MOinduced withdrawal, as observed with NX [5]. It was demonstrated in rats that subcutaneously administered NX, in contrast to quartenary opioid antagonists, decreased peripheral as well as central effects of MO-dependent rats [30].

Human studies performed in healthy volunteers and in human subjects on a methadone program showed that oral $\mathrm{N}$ methylnaltrexone shortened the orocecal transit time without producing withdrawal symptoms [12,31-33].

Quarternary opioid antagonists are suggested, according to results of subcutaneous, oral, and intravenous administration, to be unable to cross the blood-brain barrier and thus to explain the lack of opioid withdrawal symptoms under the respective treatment. Our study with MNX and NXG in the rat organ preparation indicates that both substances are unable to cross the colon-mucosal blood barrier.

However, further in vitro and in vivo studies are needed, for example, to further characterize the receptor binding affinity of NXG and to clinically elucidate the present study findings in healthy volunteers and patients.

Acknowledgment This study was supported by Grant 3200066693.01 from the Swiss National Science Foundation.

\section{References}

1. Vanegas G, Ripamonti C, Sbanotto A, De Conno F (1998) Side effects of morphine administration in cancer patients. Cancer Nurs 21:289-297

2. Grond S, Zech D, Diefenbach C, Bischoff A (1994) Prevalence and pattern of symptoms in patients with cancer pain: a prospective evaluation of 1635 cancer patients referred to a pain clinic. J Pain Symptom Manage 9:372-382

3. Schug SA, Zech D, Grond S, Jung H, Meuser T, Stobbe B (1992) A long-term survey of morphine in cancer pain patients. J Pain Symptom Manage 7:259-266
4. Holmes S (1989) Use of a modified symptom distress scale in assessment of the cancer patient. Int J Nurs Stud 26:69-79

5. Simpkins JW, Smulkowski M, Dixon R, Tuttle R (1988) Evidence for the delivery of narcotic antagonists to the colon as their glucuronide conjugates. J Pharmacol Exp Ther 244:195-205

6. Pappagallo M (2001) Incidence, prevalence, and management of opioid bowel dysfunction. Am J Surg 182:11S-18S

7. Fallon MT, Hanks GW (1999) Morphine, constipation and performance status in advanced cancer patients. Palliat Med 13:159-160

8. De Luca A, Coupar IM (1996) Insights into opioid action in the intestinal tract. Pharmacol Ther 69:103-115

9. Porreca F, Cowan A, Raffa RB, Tallarida RJ (1983) Ketazocines and morphine: effects on gastrointestinal transit after central and peripheral administration. Life Sci 32:1785-1790

10. Galligan JJ, Burks TF (1983) Centrally mediated inhibition of small intestinal transit and motility by morphine in the rat. $\mathrm{J}$ Pharmacol Exp Ther 226:356-361

11. Manara L, Bianchi G, Ferretti P, Tavani A (1986) Inhibition of gastrointestinal transit by morphine in rats results primarily from direct drug action on gut opioid sites. J Pharmacol Exp Ther 237:945949

12. Yuan CS, Foss JF, Osinski J, Toledano A, Roizen MF, Moss J (1997) The safety and efficacy of oral methylnaltrexone in preventing morphine-induced delay in oral-cecal transit time. Clin Pharmacol Ther 61:467-475

13. Bartlett SE, Dodd PR, Smith MT (1994) Pharmacology of morphine and morphine-3-glucuronide at opioid, excitatory amino acid, GABA and glycine binding sites. Pharmacol Toxicol 75:7381

14. Christrup LL (1997) Morphine metabolites. Acta Anaesthesiol Scand 41:116-122

15. Ulens C, Baker L, Ratka A, Waumans D, Tytgat J (2001) Morphine6beta-glucuronide and morphine-3-glucuronide, opioid receptor agonists with different potencies. Biochem Pharmacol 62:12731282

16. Meunier A, Latremoliere A, Mauborgne A, et al. (2005) Attenuation of pain-related behavior in a rat model of trigeminal neuropathic pain by viral-driven enkephalin overproduction in trigeminal ganglion neurons. Mol Ther 11:608-616

17. Comert M, Sipahi EY, Ustun H, et al. (2005) Morphine modulates inducible nitric oxide synthase expression and reduces pulmonary oedema induced by alpha-naphthylthiourea. Eur J Pharmacol 511:183-189

18. Lewanowitsch T, Irvine RJ (2002) Naloxone methiodide reverses opioid-induced respiratory depression and analgesia without withdrawal. Eur J Pharmacol 445:61-67

19. Maslov LN, Lishmanov YB, Solenkova NV, Gross GJ, Stefano GB, Tam SW (2003) Activation of peripheral delta opioid receptors eliminates cardiac electrical instability in a rat model of postinfarction cardiosclerosis via mitochondrial ATP-dependent $\mathrm{K}+$ channels. Life Sci 73:947-952

20. Menendez L, Lastra A, Meana A, Hidalgo A, Baamonde A (2005) Analgesic effects of loperamide in bone cancer pain in mice. Pharmacol Biochem Behav 81:114-121

21. Herrmann C, Cuber JC, Dakka T, Bernard C, Chayvialle JA (1991) Bombesin potentiates taurocholic acid-induced neurotensin release in rats. Endocrinology 128:2853-2857

22. Herrmann-Rinke C, Eissele R, Arnold R, Goke B (1996) Studies on the viability of the isolated vascularly perfused rat colon. Digestion 57:349-355

23. Reber PU, Patel AG, Lewis MP, Ashley SW, Reber HA (1998) Stenting does not decompress the pancreatic duct as effectively as surgery in experimental chronic pancreatitis. Surgery 124:561567

24. Scheurer U, Drack E, Halter F (1985) Cyclooxygenase inhibitors affect Met-enkephalin- and acetylcholine-stimulated motility of the isolated rat colon. J Pharmacol Exp Ther 234:742-746 
25. Scheurer U, Wenger F, Caliezi A, Drack E, Varga L, Halter F (1990) Different motor actions of dynorphins and nonpeptide kappa opioid receptor agonists in the isolated rat colon. J Pharmacol Exp Ther 252:1324-1330

26. Lenard L Jr., Halmai V, Bartho L (1999) Morphine contracts the guinea pig ileal circular muscle by interfering with a nitric oxide mediated tonic inhibition. Digestion 60:562-566

27. Meissner W, Schmidt U, Hartmann M, Kath R, Reinhart K (2000) Oral naloxone reverses opioid-associated constipation. Pain $84: 105-109$

28. Sykes NP (1996) An investigation of the ability of oral naloxone to correct opioid-related constipation in patients with advanced cancer. Palliat Med 10:135-144

29. Jurna I, Kaiser R, Kretz O, Baldauf J (1992) Oral naloxone reduces constipation but not antinociception from oral morphine in the rat. Neurosci Lett 142:62-64
30. Bianchi G, Fiocchi R, Tavani A, Manara L (1982) Quaternary narcotic antagonists' relative ability to prevent antinociception and gastrointestinal transit inhibition in morphine-treated rats as an index of peripheral selectivity. Life Sci 30:1875-1883

31. Yuan CS, Foss JF, O'Connor M, Toledano A, Roizen MF, Moss J (1996) Methylnaltrexone prevents morphine-induced delay in oral-cecal transit time without affecting analgesia: a double-blind randomized placebo-controlled trial. Clin Pharmacol Ther 59:469475

32. Yuan CS, Foss JF, O’Connor M, Osinski J, Roizen MF, Moss J (1999) Effects of intravenous methylnaltrexone on opioid-induced gut motility and transit time changes in subjects receiving chronic methadone therapy: a pilot study. Pain 83:631-635

33. Yuan CS, Foss JF, O'Connor M, et al. (2000) Methylnaltrexone for reversal of constipation due to chronic methadone use: a randomized controlled trial. JAMA 283:367-372 